The Astrophysical Journal, 492:778-781, 1998 January 10

(c) 1998. The American Astronomical Society. All rights reserved. Printed in U.S.A.

\title{
THE ZERO POINT OF EXTINCTION TOWARD BAADE'S WINDOW FROM K GIANTS
}

\author{
Andrew Gould, ${ }^{1}$ Piotr Popowski, And Donald M. Terndrup \\ Department of Astronomy, The Ohio State University, Columbus, OH 43210; \\ gould@astronomy.ohio-state.edu, popowski@astronomy.ohio-state.edu, \\ terndrup@astronomy.ohio-state.edu \\ Received 1997 May 9; accepted 1997 August 22
}

\begin{abstract}
We measure the zero point of the Stanek extinction map by comparing the observed $(V-K)$ colors of $206 \mathrm{~K}$ giant stars with their intrinsic $(V-K)_{0}$ colors as derived from their $\mathrm{H} \beta$ indices. We find that the zero point of the Stanek map should be changed by $\Delta A_{V}=-0.10 \pm 0.06 \mathrm{mag}$, obtaining as a bonus a threefold reduction of the previous statistical error. The most direct way to test for systematic errors in this determination would be to conduct a parallel measurement based on the $(V-K)$ colors of type $a b$ RR Lyrae stars.
\end{abstract}

Subject headings: dust, extinction - Hertzsprung-Russell diagram — stars: late-type stars: variables: other (RR Lyrae)

\section{INTRODUCTION}

Baade's window, $(\ell, b) \sim\left(1^{\circ},-4^{\circ}\right)$, has been an important laboratory for the study of bulge populations. The key features of Baade's window that made it such a focus of early work are its relatively low extinction $\left(A_{V} \sim 1.5\right)$ and the presence of NGC 6522, which provides an opportunity to measure that extinction. The value of this window increased significantly when Stanek (1996) constructed a detailed extinction map with $30^{\prime \prime}$ resolution by applying the method of Woźniak \& Stanek (1996) to observations by the Optical Gravitational Lensing Experiment (Szymański \& Udalski 1993; Udalski et al. 1993) over a 40' square field. It is now possible to deredden stellar samples on almost a star-by-star basis in this field. Stanek (1996) estimates the error in differential extinction to be $\sim 0.1 \mathrm{mag}$ in $A_{V}$ but notes that the errors in the absolute extinction are dominated by the zero-point error, $0.20 \mathrm{mag}$. For many applications, such as the interpretation of color-magnitude diagrams of bulge field stars and of the cluster NGC 6522 or the measurement of distances using RR Lyrae stars or other tracers, the determination of the zero point is crucial.

Here we argue that the best way to estimate the zero point for the $A_{V}$ map is to measure $\Delta E(V-K)_{i}$ for an ensemble of stars $i=1 \ldots n$, defined by

$$
\Delta E(V-K)_{i}=(V-K)_{i}-(V-K)_{0, i}-(1-\alpha) A_{V, i}^{\text {Stanek }},
$$

where $(V-K)_{i}$ is the observed color of the star, $(V-K)_{0, i}$ is its predicted unreddened color, $A_{V, i}^{\text {Stanek }}$ is the visual extinction at the position of the star in the Stanek (1996) map, and $\alpha$ is the ratio of the extinction in the $K$ and $V$ bands, assumed to be (Rieke \& Lebofsky 1985)

$$
\alpha \equiv \frac{A_{K}}{A_{V}}=0.11 .
$$

The correction to the zero point of the Stanek (1996) map is then given by

$$
\Delta A_{V}=\frac{\langle\Delta E(V-K)\rangle}{1-\alpha},
$$

where $\langle\Delta E(V-K)\rangle$ is a suitably weighted average of equation (1) over the sample. We then apply this method to

\footnotetext{
${ }^{1}$ Alfred P. Sloan Foundation Fellow.
}

the data of Terndrup, Sadler, \& Rich (1995; hereafter TSR) and find

$$
\Delta A_{V}=-0.10 \pm 0.06 .
$$

That is, the $A_{V}$ values of stars in Baade's window are on average 0.10 mag lower than previously believed.

\section{THE STANEK EXTINCTION MAP}

Stanek (1996) measured the mean position of the red giant clump on the color-magnitude diagram $(\langle V\rangle$, $\langle V-I\rangle)$ as a function of position on the sky $(x, y)$. He then estimated the differential total and differential selective extinctions,

$$
\begin{aligned}
A_{V}(x, y) & =\langle V\rangle(x, y)+C_{V}, \\
E(V-I)(x, y) & =\langle V-I\rangle(x, y)+C_{V-I},
\end{aligned}
$$

with the constants, $C_{V}$ and $C_{V-I}$, being undetermined. He found a very strong empirical correlation between $A_{V}$ and $E(V-I)$,

$$
A_{V}(x, y) \simeq 2.49 E(V-I)(x, y)+C .
$$

On physical grounds, $C=0$. This left one constant, either $C_{V}$ or $C_{V-I}$, to be determined.

Stanek (1996) then determined the overall zero point using the measurement $E(V-I)=0.59 \pm 0.08$ made by TSR in a subregion of Baade's window, Blanco region A (Blanco, McCarthy, \& Blanco 1984). That is, he set the zero point so that the average of $E(V-I)$ over this region of his map reproduced the TSR value. He then used equation (6) (with $C=0$ ) to establish the zero point of the $A_{V}$ map; i.e., he set the mean extinction of region A to be $\left\langle A_{V}\right\rangle=0.59$ $\times 2.49=1.47$. The formal error in this determination is therefore $0.08 \times 2.49=0.20$.

\section{PREVIOUS APPROACHES}

With the exception of TSR, all previous determinations of the extinction toward Baade's window have been made by measuring the selective extinction $E(B-V)$ and then multiplying by an assumed ratio of total to selective extinction $R_{V}=A_{V} /[E(B-V)]$ (Arp 1965; van den Bergh 1971; Walker \& Mack 1986; Terndrup \& Walker 1994). There are several major disadvantages to this approach. First, $R_{V} \sim 3$ is rather large, and the statistical error in $E(B-V)$ (usually estimated to be $\geq 0.03$ ) is multiplied by this factor when 
estimating the error in $A_{V}$. Second, $R_{V}$ varies along different lines of sight, so for any particular line of sight for which it is not actually measured (e.g., Baade's window) the precision of the estimate is no better than 7\%. Hence, the statistical error alone for $A_{V}$ is more than $0.12 \mathrm{mag}$. Finally, there are systematic errors arising from uncertainties in the intrinsic $B-V$ colors of stars used to estimate $E(B-V)$. While the intrinsic color of extremely hot stars (in the Rayleigh-Jeans limit) is known from fundamental physics, there are no such stars lying beyond the dust column in this direction. Hence, one must use cooler stars whose $B-V$ colors are sensitive functions of temperature, metallicity, and perhaps other factors. The standard approach is to find local analogs of the program stars and directly measure their colors, but systematic errors may arise from any unrecognized differences between these two groups of stars. As always, it is difficult to determine the size of the systematic errors, but one can gain a sense of their magnitude by comparing the $E(B-V)_{0}=0.45 \pm 0.04$ derived by van den Bergh (1971) from three different methods based on cool stars $(\mathrm{K}$ and $\mathrm{M}$ giants) with the $E(B-V)_{0}=0.60 \pm 0.03$ derived by Walker $\&$ Mack (1986) using relatively hot stars (RR $a b$ stars). Here the subscript " 0 " means " reduced to zero color," using the prescription adopted by TSR from Dean, Warren, \& Cousins (1978).

TSR pioneered a radically different approach, although they did not call explicit attention to this fact. They measured

$$
E(V-K)=1.23 \pm 0.08
$$

for the Blanco A region by comparing the $\mathrm{H} \beta \lambda 4861$ (Faber et al. 1985) index as a function of calculated $V-K$ color to that observed for bright $\mathrm{K}$ giants in the solar neighborhood. They then inferred (but did not explicitly write),

$$
A_{V}=\frac{E(V-K)}{1-\alpha}=1.38 \pm 0.09 .
$$

While this approach is formally identical to the previous one (measurement of a selective extinction and conversion to a total extinction), it is potentially more accurate than using $E(B-V)$ because the extrapolation to total visual extinction is small (a factor of 1.12 instead of 3), and therefore the error in $A_{V}$ is only slightly bigger than the error in $E(V-K)$. TSR then used this measurement to infer $E(B-V)_{0}=0.47 \pm 0.04$ and $E(V-I)=0.59 \pm 0.08$. To obtain these quantities, they had to employ estimates of the ratios of total to selective extinctions that account for the proportionately larger error bars compared to those in equations (7) and (8). In particular, TSR assumed

$$
A_{V}=2.33 E(V-I),
$$

where the coefficient is considerably lower than empirical value for Baade's window (eq. [6]) used by Stanek (1996). By fixing the zero point according to TSR's $E(V-I)$ rather than to TSR's more directly determined (but unreported) $A_{V}$, Stanek (1996) therefore overestimated the visual extinction by $(2.49-2.33) \times 0.59=0.09 \mathrm{mag}$ and also overestimated the uncertainty. From this simple argument, we therefore derive a naive correction to the Stanek (1996) extinction map,

$$
\Delta A_{V}=-0.09 \pm 0.09 \quad \text { (naive) } .
$$

\section{NEW DETERMINATION}

However, rather than simply adopting the correction given by equation (10), we prefer for several reasons to make a complete redetermination of the zero point of the Stanek (1996) extinction map. First, Stanek (1996) compared the TSR extinction for the whole of region $A$ with the mean extinction of the subregion of region A that is covered by his map. About 35\% of the TSR stars lie within 2' of NGC 6522 and so are excluded from the map. One should therefore compare the mean extinction of the TSR stars lying within the map with the mean extinction predicted by the map for their positions; otherwise systematic trends of extinction with position could affect the result. Second, TSR measured and reported the mean extinctions in region $\mathrm{A}$ and in regions $\mathrm{B}$ and $\mathrm{C}$ separately. They did so because these areas have different average extinctions (Blanco et al. 1984), so it would degrade the information content of their results to combine the two. However, for purposes of measuring the offset to the Stanek (1996) map, the fact that different regions have different extinctions is irrelevant. The only concern is to measure the difference in the observed extinction at each point from that predicted by the map. Including all three regions approximately triples the sample and correspondingly reduces the statistical errors. Third, TSR used the relatively crudely determined $\mathrm{H} \beta /(V-K)_{0}$ relation of Faber et al. (1985). A much more sophisticated polynomial relation is available from Gorgas et al. (1993, see especially Table 6 and eq. [5]):

$$
\begin{aligned}
& \mathrm{H} \beta= 8.2261-5.9295(V-K)_{0}+0.52968(\log g) \\
&-0.048352(\log g)^{2}-0.23695(\log g)(V-K)_{0} \\
&+ 1.8169(V-K)_{0}^{2}-0.19721(V-K)_{0}^{3},
\end{aligned}
$$

where we have ignored the terms in $[\mathrm{Fe} / \mathrm{H}]$ since they are small and since $\langle[\mathrm{Fe} / \mathrm{H}]\rangle \sim 0$ for the sample in any case.

To carry out our analysis, we first restrict the sample to the 209 stars with

$$
2.1 \leq(V-K)_{*} \leq 3.0,
$$

where

$$
(V-K)_{*} \equiv(V-K)-(1-\alpha)\left(A_{V}^{\text {Stanek }}+\Delta A_{V, *}\right)
$$

is our final best-fit estimate of $(V-K)_{0}$ as determined from our final best-fit offset to the Stanek (1996) map (eq. [4]). The upper limit is chosen to exclude $\mathbf{M}$ giants that have TiO bands that influence the $\mathrm{H} \beta$ index. Gorgas et al. (1993) also excluded these stars when they derived equation (11). We note, however, that if we extend the sample to the 239 with $(V-K)_{*}<3.8$ (the range of the Gorgas et al. 1993 data), the results change by less than $0.01 \mathrm{mag}$. The lower limit in equation (12) is justified below.

To obtain $V-K$ from the $V$ and $I$ measurements, we slightly modify the procedure of TSR. TSR converted from $V-I$ to $V-K$ colors based on the extremely tight quadratic color-color relation obtained for a patch of Baade's window by Tiede, Frogel, \& Terndrup (1995). We modify this procedure by using the Stanek (1996) differential map to transform each $V-I$ from its observed value to the value it would have if it lay in the Tiede et al. (1995) region and then use the same relative extinction to transform the inferred $V-K$ back to the star's actual position. In practice, the individual corrections generated by this procedure are small, typically $<0.01 \mathrm{mag}$, because the color-color track is 


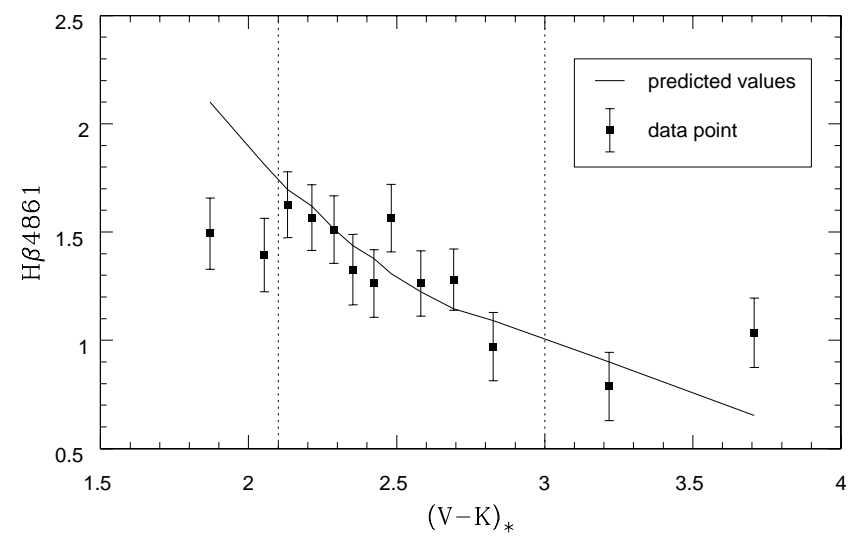

FIG. 1.-Observed $\mathrm{H} \beta$ index (solid squares) compared to the values predicted (solid curve) from the relation of Gorgas et al. (1993). The prediction is mainly a function of $(V-K)_{*}$, which is the best-fit estimate of $(V-K)_{0}$ using the observed $V-K$ color, the Stanek (1996) extinction for the position of each star, and the overall offset given by eq. (4). The points are binned by $\sim 23$ stars, and the error bars indicate the standard error of the mean based on the individual errors reported by TSR. The stars with $(V-K)_{*}>3.0$ (right-hand dashed line) were excluded from the fit because they are $\mathrm{M}$ giants that are affected by TiO bands and because Gorgas et al. (1993) excluded such stars when they determined eq. (11). The stars with $(V-K)_{*}<2.1$ (left-hand dashed line) were excluded from the fit because they are contaminated with foreground stars and hence fall systematically below the predicted values (see text).

almost parallel to the reddening vector. The net effect on the final result is $\ll 0.01 \mathrm{mag}$.

We estimate the surface gravity $g$ from the inverse square of the star's effective radius in $K$ band,

$$
\log \frac{g}{g_{\odot}}=\log \frac{S}{S_{\odot}}+0.4\left(K-\alpha A_{V}-M_{K, \odot}-\mu\right),
$$

where $S \propto\left[\exp \left(h c / \lambda_{K} k T\right)-1\right]^{-1}$ is the blackbody surface brightness in the $K$ band $\left(\lambda_{K}=2.2 \mu \mathrm{m}\right)$ for an assumed temperature $\quad T=8520-2230(V-K)_{0}+267(V-K)_{0}^{2}$, obtained by fitting the values given in Table 4 of Ridgway et al. (1980). Here $\mu=14.5$ is the adopted distance modulus to the Galactic center and $M_{K, \odot}=3.3$.

For each trial value of $\Delta A_{V}$, we deredden the "observed" $V-K$ color (inferred from $V-I$ ) and $K$ magnitude of each star, use these to estimate its temperature and surface gravity, and finally predict $\mathrm{H} \beta$ from equation (11). We form $\chi^{2}\left(\Delta A_{V}\right)$ from the difference of the observed $\mathrm{H} \beta$ and these predictions divided by the errors as reported by TSR. The best-fit $\Delta A_{V}$ and its errors are determined from this function.

We find that in the adopted interval, $2.1 \leq(V-K)_{*} \leq$ 3.0, the predicted and observed $\mathrm{H} \beta$ indices (weighted by the observational errors) are in good overall agreement with $\chi^{2}=179$ for 208 degrees of freedom. However, the observations deviate markedly from the predictions for $(V$ $-K)_{*}<2.1$ (see Fig. 1, below). These bluer stars are mostly $\mathrm{G}$ giants and subgiants. The original sample from which TSR drew their stars was selected primarily for propermotion studies and hence was composed of preferentially brighter stars. The intrinsically fainter $\mathrm{G}$ stars are therefore likely to be foreground disk stars. From equations (11) and (14), we find that if one of these star lies $\Delta \mu$ in the foreground, $\mathrm{H} \beta$ will be overestimated by $\sim 0.1 \Delta \mu$. Thus, this selection effect can explain at least some of the observed deviation. In any event, these stars clearly differ from the bulk of the $\mathrm{K}$ giants (which dominated the fit by Gorgas et al. 1993), and we therefore restrict the sample to $(V-K)_{*} \geq$ 2.1.

We now consider various systematic effects. First, there are several assumptions that affect the results through the estimate of the surface gravity. For example, we find that if the adopted distance to the Galactic center is increased by $\Delta \mu$, then the surface gravities are decreased, implying that $\Delta A_{V}$ is also decreased (becomes more negative) by $-0.11 \Delta \mu$. Similarly, if the mean mass of the stars is larger than $1 M_{\odot}$ (the value that we implicitly assumed in writing eq. [14]), then $\Delta A_{V}$ is increased by $0.10 \Delta \ln M$. If the effective radius at which surface gravities are measured differs from that of the $K$-band photosphere, $\Delta A_{V}$ is changed by $-0.20 \Delta \ln R$. If the adopted temperatures are on average different from the true ones, $\Delta A_{V}$ is changed by $0.19 \Delta \ln T$. Plausible adjustments for each of these factors are therefore likely to affect $\Delta A_{V}$ by $\lesssim 0.01 \mathrm{mag}$.

Gorgas et al. (1993) report a scatter in their fit to the $\mathrm{H} \beta$ index of 0.28 . We estimate that this scatter causes an uncertainty in the zero point of equation (11) of $\sim 0.025$, which translates directly into an uncertainty in $\Delta A_{V}$ of $0.03 \mathrm{mag}$.

Next, we break the sample into two subsamples, one of stars in region A (where TSR found $A_{V}=1.38$ ) and one of stars in regions B and $\mathrm{C}$ (where they found $A_{V}=1.55$ ). We obtain separate fits of $\Delta A_{V}=-0.07 \pm 0.09$ and $\Delta A_{V}=$ $-0.13 \pm 0.06$, respectively, which are consistent at the $1 \sigma$ level.

Finally, we divide the sample into two subsamples, according to their angular distance $\theta$ from the center of NGC 6522. The inner group $\left(2^{\prime} .0<\theta<2{ }^{\prime} .5\right)$ has 49 stars, and the outer group $(\theta>2.5)$ has 160 stars. We find values of $\Delta A_{V}=-0.28 \pm 0.12$ and $\Delta A_{V}=-0.06 \pm 0.06$, respectively. That is, they are inconsistent at the $1.6 \sigma$ level. This is somewhat worrisome because it may indicate that the inner group is affected in some way by proximity to the cluster. We investigate the following possible effects. First, Stanek (1996) reports that the extinction values close to NGC 6522 and NGC 6528 are systematically lower than in surrounding regions, leading him to believe that he may have underestimated it. (In the method of Woźniak \& Stanek 1996, subregions are rank ordered in extinction according to their relative stellar density. Hence the presence of a cluster would tend to increase the stellar density, causing one to underestimate the extinction. This was the primary reason for excluding the region $\theta<2^{\prime}$ from the map.) It is possible that this effect extends beyond 2 . However, the sign of the effect is wrong to explain the difference between the two subsamples. It is possible that contamination by cluster stars generates some other effect that has the correct sign. We perform the following tests to search for cluster contaminants. First we search for an excess of stars in the underlying sample (which reaches as close as $\theta \sim 1^{\prime}$ from the cluster center) with radial velocities that are consistent with the cluster velocity, $\sim-25 \mathrm{~km} \mathrm{~s}^{-1}$ (Rich 1990; Smith, Hesser, \& Shawl 1976), both in the sample as a whole and as a function of $\theta$. If contamination by the cluster were significant, one would expect an excess, especially at small radii. None is detected. Next we conduct a test that is sensitive to even lower levels of contamination: we plot the proper motions of all stars having radial velocities consistent with cluster membership. If even a small subset of these are in the cluster, the proper-motion diagram should show a clump. We detect a common proper-motion clump of eight stars. 
We will report elsewhere on this measurement of the proper motion of NGC 6522 (Terndrup et al. 1998). For present purposes, we note that three of the eight stars are part of our sample, all three being in the inner annulus. We exclude these, leaving a sample of 206 stars. The estimates for $\Delta A_{V}$ in the inner and outer annuli are then $-0.25 \pm 0.12$ and $\Delta A_{V}=-0.06 \pm 0.06$, respectively, a $1.4 \sigma$ difference. Since contamination by the cluster has been eliminated, this difference should be regarded as a normal statistical fluctuation, and we therefore include the entire remaining sample of 206 stars and find

$$
\Delta A_{V}=-0.10 \pm 0.05 \quad \text { (internal error) } .
$$

We then add in quadrature the external calibration error of 0.03 mag intrinsic to the Gorgas et al. relation (11) to obtain equation (4). Figure 1 shows the mean predicted and observed $\mathrm{H} \beta$ indices (weighted by the observational errors) for the overall best fit, binned by $(V-K)_{*}$. We find $\chi^{2}=176$ for 205 degrees of freedom.

For completeness, we note that had we adopted the relation of Faber et al. (1985) in place of equation (11) from Gorgas et al. (1993), $\Delta A_{V}$ would decrease from -0.10 to -0.11 . 5. FUTURE TESTS

Of course, the most important potential source of systematic errors is not probed by the tests of $\S 4$ : the possibility that the stars in Baade's window differ systematically in some unknown way from the local stars upon which equation (11) is based. Ultimately, the only way to test for this effect is to make an independent determination of $E(V-K)$ on a substantially different set of stars. The obvious choice for this test is type $a b$ RR Lyrae stars. First, the comparison between RR $a b$ stars and cool giants shows the largest discrepancy of all determinations based on $E(B-V)$, so it is important to see if this discrepancy persists for $E(V-K)$. Second, because of the relatively narrow range of RR $a b$ colors and the accuracy of the Stanek (1996) map, we estimate that each star should provide a statistically independent estimate of the zero point accurate to 0.15 mag. Since there are more than 50 such stars in the region, the limit for this method is set by the size of the calibrating sample (17) observed by Jones et al. (1992). We estimate this limiting uncertainty to be only $\pm 0.03 \mathrm{mag}$. Work is in progress to apply this method.

Work by A. G. and P. P. was supported in part by grant AST 94-20746 from the NSF. Work by D. M. T. was supported in part by grant AST 95-28227 from the NSF.
Arp, H. 1965, ApJ, 141, 43

Blanco, V. M., McCarthy, M. F., \& Blanco, B. M. 1984, AJ, 89, 636

Dean, J. F., Warren, P. R., \& Cousins, A. W. J. 1978, MNRAS, 183, 569

Faber, S. M., Friel, E. D., Burstein, D., \& Gaskell, C. M. 1985, ApJS, 57, 711

Gorgas, J., Faber, S. M., Burstein, D., González, J., Courteau, S., \& Prosser, C. 1993, ApJS, 86, 153

Jones, R. V., Carney, B. W., Storm, J., \& Latham, D. W. 1992, ApJ, 386, 646 Rich, R. M. 1990, ApJ, 362, 604

Ridgway, S. T., Joyce, R. R., White, N. M., \& Wing, R. F. 1980, ApJ, 235, 126

Rieke, G. H., \& Lebofsky, M. J. 1985, ApJ, 288, 618

\section{REFERENCES}

Smith, M. G., Hesser, J. E., \& Shawl, S. J. 1976, ApJ, 206, 66

Stanek, K. Z. 1996, ApJ, 460, L37

Szymański, M., \& Udalski, A. 1993, Acta Astron., 43, 91

Terndrup, D. M., Popowski, P., Gould, A., Rich, R. M., \& Sadler, E. M. 1998 , in preparation

Terndrup, D. M., Sadler, E. M., \& Rich, R. M. 1995, AJ, 110, 1774 (TSR)

Terndrup, D. M., \& Walker, A. R. 1994, AJ, 107, 1786

Tiede, G. P., Frogel, J. A., \& Terndrup, D. M. 1995, AJ, 110, 2788

Udalski, A., et al. 1993, Acta Astron., 44, 165

van den Bergh, S. 1971, AJ, 76, 1082

Walker, A. R., \& Mack, P. 1986, MNRAS, 220, 69

Woźniak, P. R., \& Stanek, K. Z. 1996, ApJ, 464, 233 